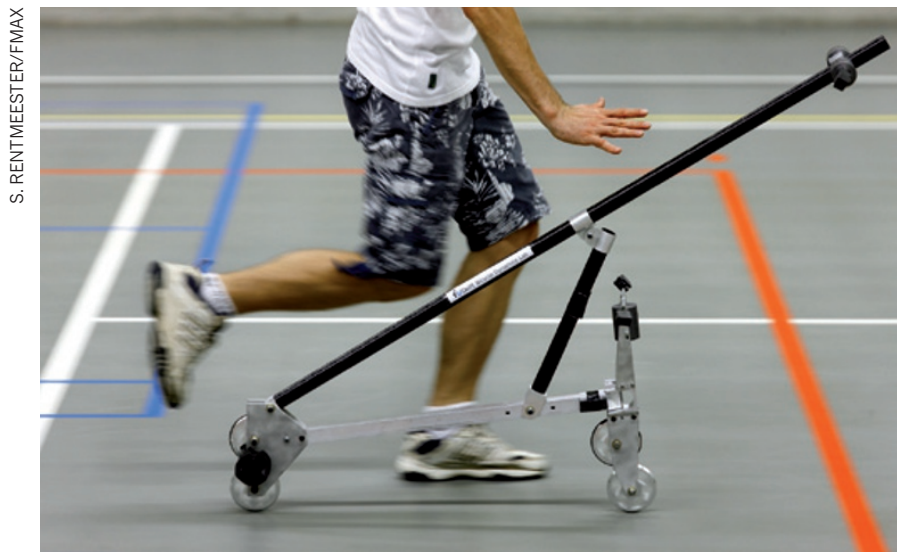

MECHANICS

\section{A stable, riderless bicycle}

A riderless bicycle can travel for some distance without falling - an ability generally assumed to be due to the gyroscopic forces of the spinning front wheel keeping it upright. But this is not the whole story, according to a paper by Andy Ruina of Cornell University in Ithaca, New York, and his colleagues. Following a theoretical model, the team built a bicycle (pictured) in which the gyroscopic forces were cancelled by a second set of wheels, off the ground, that spun in the reverse direction.

The authors found that the bike was relatively stable at speeds of more than 2 metres per second, showing that gyroscopic forces are not necessary for bike stability. In the case of their custom bike, the low steering assembly handlebars, fork and front wheel - fell faster than the rest of the bike, tending to steer the bike in the direction of the fall, just as a rider would do. Science 332, 339-342 (2011)

\section{BEHAVIOUR}

\section{Diversity beats ability}

Can you guess how many marbles are in a jar? Maybe you are a savant or maybe not; either way, odds are that, between them, a group of people will beat your guess.

Stefan Krause at the Lübeck University of Applied Sciences in Germany and his colleagues asked visitors to a museum to play the marble guessing game, and collected more than 2,000 guesses. When they made random groups out of these results, they found that the average guess of groups with 40 or more members was better than the best quarter of individual guesses, which implies that large groups of average ability are smarter than individual stars. The result suggests that, like schooling fish or swarming bees, humans can make fruitful use of collective decision-making, and that groups might outperform individuals of high ability hinting at a selection pressure for diverse personalities.

Anim. Behav. 81, 941-948 (2011)

\section{CANCER BIOLOGY}

\section{Laying siege to cancer}

Tumours can be shrunk by inhibiting a naturally occurring transcription factor called Myc to make the tumour microenvironment inhospitable to invasive cells.

Gerard Evan, now at the University of Cambridge, UK, and his team tested the effects of Myc suppression in a mouse model of pancreatic cancer. Inactivating Myc in tumours incapacitated the healthy cells that

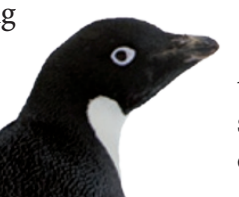

The most viewed papers in science

\title{
Sleep deprivation: a risky business
}

\section{AHGHLY READ \\ on jneurosci.org in March}

What do stock traders, doctors and soldiers have in common? Each often needs to make important decisions on little sleep. Michael Chee of the Duke-NUS

Graduate Medical School in Singapore and his colleagues used brain imaging to study how sleep deprivation affects decisionmaking. They scanned the brains of healthy adults performing a complicated gambling task. When the volunteers had stayed up all night, their strategy shifted towards seeking bigger gains over protecting against losses. The shift in behaviour correlated with changes in brain activity in regions previously associated with reward evaluation and regret.

Interestingly, the shift in strategy did not correlate with vigilance, suggesting that although stimulants such as caffeine might make sleepy people more alert, they do not improve decision-making abilities.

J. Neurosci. 31, 3712-3718 (2011)

support cancer cells. This resulted in collapsed blood vessels and reduced inflammatory responses, leading to oxygen starvation and death for tumour cells.

The fact that tumour cell death occurred after microenvironment collapse, even if Myc was inhibited only in cancer cells, suggests that the transcription factor functions as a communicator between tumours and their surroundings. And, as an essential contributor to tumour growth and maintenance, Myc is an attractive therapeutic target - especially because most cells can't wiggle around Myc suppression.

Genes Dev. doi:10.1101/

gad.2038411 (2011)

\section{CLIMATE CHANGE}

\section{Sea ice penguin theory sinks}

As sea ice has declined off the West Antarctic Peninsula, scientists expected to see declines in ice-dwelling Adélie penguins (pictured left) and increases in chinstrap penguins (right), which forage in ice-free waters. However, Wayne Trivelpiece and his colleagues at the US National Marine Fisheries Service in La Jolla, California, say there is now "overwhelming evidence" that both populations are declining in the region.

The authors' data, which cover populations at two sites over 30 years, suggest that sea-ice losses resulting from climate change have reduced the availability of Antarctic krill, the prey of both birds. If temperatures rise in future, sea ice and krill will decline further, and both species of penguin are likely to follow. Proc. Natl Acad. Sci. USA doi:10.1073/ pnas. 1016560108 (2011)
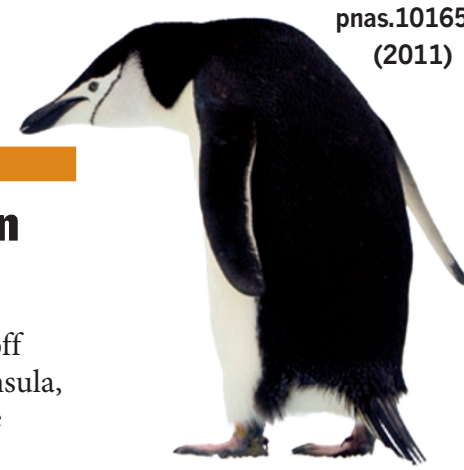

$\rightarrow$ NATURE.COM

For the latest research published by Naturevisit:

www.nature.com/latestresearch 